\title{
Patient preferences for adherence to treatment for osteoarthritis: the MEdication Decisions in Osteoarthritis Study (MEDOS)
}

Tracey-Lea Laba ${ }^{1,2^{*}}$, Jo-anne Brien ${ }^{1,3,4}$, Marlene Fransen ${ }^{5}$ and Stephen Jan ${ }^{2,6}$

\begin{abstract}
Background: Often affecting knee joints, osteoarthritis (OA) is the most common type of arthritis and by 2020 is predicted to become the fourth leading cause of disability globally. Without cure, medication management is symptomatic, mostly with simple analgesics such as acetaminophen and non-steroidal anti-inflammatory drugs (NSAIDs), and glucosamine sulfate. Adherence to arthritis medications is generally low. Intentional non-adherence, that is deliberate decision-making about the use of analgesics, occurs in OA patients. To date, a limited number of studies have explored medication-taking decisions in people with OA nor the extent to which individuals' trade off one treatment factor for another in their decision-making using quantitative techniques. This study aimed to estimate the relative influence of medication-related factors and respondent characteristics on decisions to continue medications among people with symptomatic OA.

Methods: A discrete choice experiment (DCE) was conducted among participants attending end-of-study visits in the Long-term Evaluation of Glucosamine Sulfate (LEGS) study (ClinicalTrials.gov ID: NCT00513422). The paper-based survey was used to estimate the relative importance of seven medication specific factors (pain efficacy, mode of action, dose frequency, treatment schedule, side effects, prescription, and out-of-pocket costs) and respondent characteristics on decisions to continue medications.

Results: 188 (response rate 37\%) completed surveys were returned. Four of the seven medication factors (side effects, out-of-pocket costs, mode of action, treatment schedule) had a significant effect on the choice to continue medication; patient characteristics did not. Assuming equivalent pain efficacy and disease-modifying properties for glucosamine, the positive relative likelihood of continuing with sustained-release acetaminophen was equivalent to glucosamine. By contrast, the negative relative likelihood of NSAID continuation was mostly driven by the side effect profile. The predicted probability of continuing with glucosamine decreased with increasing out-of-pocket costs.

Conclusions: This study has characterised the complexity of medication-taking decisions that potentially underpin intentional non-adherent behaviour for people with symptomatic OA. In particular, medication risks and cost were important and ought to be borne into considerations in interpreting clinical trial evidence for practice. Ultimately addressing these factors may be the way forward to realising the full potential of health and economic benefits from the efficacious and safe use of OA medications.
\end{abstract}

Keywords: Osteoarthritis, Discrete choice experiment, Intentional medication adherence

\footnotetext{
* Correspondence: thar7910@uni.sydney.edu.au

${ }^{1}$ Faculty of Pharmacy, The University of Sydney, Camperdown, Sydney, Australia

${ }^{2}$ The George Institute for Global Health, Camperdown, Sydney, Australia

Full list of author information is available at the end of the article
} 


\section{Background}

Osteoarthritis (OA) is a musculoskeletal disease that causes chronic joint pain and reduced physical functioning. Often affecting knee joints, OA is the most common type of arthritis. By 2020, OA is predicted to become the fourth leading cause of disability globally [1].

Currently there is no known cure for OA, nor are there effective interventions to slow disease progression [2-4]. Medication management is symptomatic, mostly with simple analgesics such as acetaminophen and non-steroidal anti-inflammatory drugs (NSAIDs) [5,6]. Increasingly, glucosamine sulfate (GS) [7], is being used as a potential analgesic and disease-modifying agent [3,8-11]. In Australia, GS is considered a dietary supplement and is purchased without prescription. Unlike other OA medications, the cost of GS is not subsidised by the Australian government [12].

As occurs with most chronic conditions, adherence to arthritis medications is low [4,13-16]. Factors implicated in adherence to OA and other anti-rheumatic medications include dosing frequency [16], pain and self-efficacy levels [13], and physician trust $[4,17,18]$. Intentional nonadherence [19], that is deliberate decision-making about the use of OA medications, is reported in the literature. In particular, intentional under-dosing and rationing of analgesics occurs [20-22]. Such decisions appear to be driven by factors including the fear of addiction [20], previous medication effectiveness, and the burden and illness stigma represented by increased pill loads [21]. For NSAIDs specifically, a high level of trust in the prescribing physician influences decisions [22].

Primarily, qualitative methods have been used to investigate medication decisions in OA. Although a limited number of studies have used quantitative techniques, the extent to which individuals' trade off one treatment factor for another in decision-making about medication adherence has not been extensively studied [23-28]. Physicians and policy makers could use such information to tailor adherence support to match the preferences of OA patients.

Discrete choice experiment (DCE) is a survey methodology that can be used to elicit preferences to quantitatively determine the relative influence of factors on decision-making with regard to medication adherence $[29,30]$. Developed initially in marketing research, DCEs are used increasingly in health economics and are considered state-of-the-art in this field to elicit preferences for health services [31-35]. To date, three DCE studies have explored patient preferences for treatment factors associated with knee and/or hip osteoarthritis [23,24,28]. In these studies, both efficacy and the gastrointestinal side effects of treatment significantly impacted patient choice. However, other factors potentially relevant to OA medication adherence were not consistently included. In particular, neither the cardiovascular, hepatic or renal side effects nor the chronic or intermittent treatment scheduling of OA therapy were incorporated. Additionally, preferences about acetaminophen were omitted from one study [24].

In Australia, the LEGS (Long-term Evaluation of Glucosamine Sulfate) study was a two-year, double-blind, placebo-controlled randomised clinical trial aiming to evaluate whether the dietary supplements, GS and/or chondroitin can limit or reduce structural disease progression (cartilage loss), whilst providing pain relief, in people with osteoarthritis of the knee (ClinicalTrials.gov Identifier NCT00513422). Throughout the LEGS study, as is typically the case in clinical trials, a number of trial-related factors could potentially affect treatment decisions and adherence outside of the trial setting. Firstly, as a part of the study protocol, participants were regularly encouraged to persist with the study treatments, even in the absence of knee pain. Furthermore, study treatments were mailed to participants and provided free of charge. The use of DCEs potentially helps understand the effects of such factors, including out-of-pocket costs, beyond the clinical trial setting.

The Medication Decisions in Osteoarthritis Study (MEDOS) aimed to estimate the relative influence of different medication-related factors and respondent characteristics on decisions to continue medications among people with symptomatic OA.

\section{Methods}

A paper-based survey was given to all LEGS participants attending their end-of-study visit by a member of the LEGS research team; surveys were mailed to participants who had already completed end-of-study visits at MEDOS commencement. The survey was self-administered and completed either during the end-of-study visit or at a later date and returned via mail.

\section{Survey instrument}

The survey used a DCE approach and comprised 16 hypothetical choice tasks Additional questions about selfreported adherence to study treatment and other prescribed medications during the LEGS study [36], and an eight-item scale from the primary care assessment survey (PCAS) [37] were also included. The PCAS has been used in other adherence-related research [36], and assesses the level of trust held by a patient for his/her provider.

\section{Instrument development}

In a DCE, respondents are offered a series of hypothetical pairwise alternatives (choice set), and asked to nominate the preferred alternative. Each alternative is described by a set of factors with pre-specified levels. The levels assigned to each alternative are varied successively across each choice set $[32,38,39]$. For this study, 
factors were identified through literature review and with respect to the currently available OA treatments in Australia [3,12]. Further factor refinement occurred through survey piloting among healthcare professionals and a general population experienced with analgesic use. Seven factors considered most important through survey pre-testing and used in the final survey are summarised in Table 1, which also includes description of the levels of each factor. Extensive descriptions of the factors were included in the survey introduction. Participants were advised to contact the research team should assistance be required.

The final survey included 16 choice sets. In each, respondents were presented with hypothetical medication alternatives, 'Medication A' and 'Medication B'. To eliminate product recognition bias, brand names were not used. Respondents were asked to imagine their current pain score (as measured throughout the LEGS study) was 9 out of 10 and they were currently taking both tablets, of which their doctor was aware. Given a choice between the two medication options, respondents were asked to indicate which medication they would prefer to continue taking.

On the basis of the factors and levels listed in Table 1, an orthogonal design was generated using the choice experiment design software Ngene Version 1.0 [40]. Two survey versions were created by randomly ordering the choice sets using a random number generator [41]. The survey was pilot tested $(n=5)$ to check for any problems with interpretation and face validity; only minor changes to the layout were made.

\section{Participants}

All LEGS participants completing their end-of-study visit were eligible to participate in MEDOS. The eligibility criteria for participation in the LEGS study can be found in Additional file 1 [42].

The University of Sydney Human Research Ethics Committee (HREC 8821, amendment 4th May 2010) and the Royal Australian College of General Practitioners (NREEC 06/006, amendment 18th June 2010) approved this study.

\section{Analysis}

The background characteristics of MEDOS participants were summarised and compared with all LEGS participants attending their final follow-up visit. Additionally, self-reported adherence to study treatment, cost-related non-adherence in the past 12 months, and the transformed PCAS Physician Trust domain (maximum score 100) were summarised for MEDOS participants.

For the choice data, a panel mixed multinomial (random parameters) logit (MMNL) model was used [32,38] to investigate changes in utility (U) (i.e. preference to continue taking a medication) when the level of a factor was changed using NLOGIT Version 4.0. A higher or more positive utility indicates increased preference to continue a medication. Additional file 2 details the model form and analysis plan.

The effect on the final model of respondent characteristics was investigated by forward stepwise addition followed by backwards elimination of significant covariates. A differential out-of-pocket cost factor was investigated based on work status (employed, unemployed, retired/semi-retired), as well as healthcare concession card or private health insurance through the incorporation of cost-factor interaction terms.

From the final model, the odds ratio (OR) of each factor was calculated (i.e. $\mathrm{OR}=\exp (\beta)$ ), representing the influence of the factor on the choice to continue a

Table 1 Description of factors and levels used in the discrete choice experiment

\begin{tabular}{|c|c|c|}
\hline Factor & Description & Levels \\
\hline Pain Efficacy & What the pain can be reduced to (from 9/10) & $1,3,4,7$ \\
\hline \multirow[t]{2}{*}{ Mode of action } & How the medication works & Quick pain relief (Base) \\
\hline & & Slow Osteoarthritis \\
\hline Dose frequency & How often taken per day & 1, 3 (Base) \\
\hline \multirow[t]{2}{*}{ Treatment Schedule } & How regularly taken & When needed (Base) \\
\hline & & Daily \\
\hline Cost & Cost to YOU every month & $\$$ AUS 5, 20, 3550 \\
\hline \multirow[t]{2}{*}{ Prescription } & Prescription/purchase restrictions & Yes: (pharmacy with prescription) \\
\hline & & No: (pharmacy, health food store or supermarket without prescription) (Base) \\
\hline \multirow[t]{4}{*}{ Side effects } & Possible side effects of the medication & No side effects (Base) \\
\hline & & Drowsy/constipated \\
\hline & & Heartburn/reflux, stomach ulcers \\
\hline & & High blood pressure, heart/kidney/liver problems \\
\hline
\end{tabular}


medication. Odds ratios were also calculated for the medication profiles for GS, sustained-release acetaminophen, and selective and non-selective NSAIDs by inputting the factor levels outlined in Table 2. This represents the relative likelihood of continuing each medication profile: an OR greater than 1 represents an increased preference to continue taking medication.

The willingness to accept (WTA) for each factor was estimated by taking the marginal rate of substitution between the factor and cost $\left(\beta_{\text {factor }} / \beta_{\text {cost }}\right)$ [43]. This describes the amount of money respondents believe compensates for a given change in the factor.

The relative importance of factors and their levels was also investigated [34]. This reflects the extent to which the difference between the best and worst levels of each characteristic drives the decision to continue taking a medication.

Finally, the predicted probability of continuing GS was calculated using the factor levels outlined in Table 2 [44]. As the cost of GS is variable within Australia, a cost sensitivity analysis was conducted for the predicted probability.

\section{Results}

503 LEGS participants attended the end-of-study visits; 59 participants had already attended the end-of-study visit at MEDOS commencement and were mailed a survey. The remaining participants were given a survey at the end-of-study visit.

188 (response rate 37\%) completed surveys were returned. Table 3 displays the background characteristics for all LEGS participants attending their end-of-study visit, and the subset of these participants completing the MEDOS study. With the exception of a lower proportion of people taking "when required" medications in the MEDOS group, there does not appear to be evidence for difference.

For the MEDOS participants, self-reported adherence to the study treatment throughout the LEGS study was generally high, ( $47 \%$ reporting $100 \%$ adherence). The lowest adherence rate reported was $75 \%$. For other medications, $24 \%$ of participants reported intentionally stopping or altering the dose during the previous 12 months. In general, MEDOS study participants had a high level of trust in their primary care physician (median PCAS score 75 out of 100).

\section{DCE results}

Table 4 shows the results of the DCE. An estimated OR of 0.90 implies that changing the treatment schedule from "when required" to "daily treatment" decreases the likelihood of continuing a medication by $10 \%$, if all other factors are held equal. Likewise, an OR of 0.09 implies that changing from a medication with no side effects to one that may cause high blood pressure, heart, kidney, or liver side effects decreases the likelihood of continuing a medication by $91 \%$.

Four of the seven factors had a significant effect on the choice to continue a medication: out-of-pocket costs, side effects, mode of action, and treatment schedule. Pain efficacy, dose frequency, and whether one's access to the medication was restricted through prescription and place of purchase did not significantly influence medication choice. The signs of all significant parameters were in the expected direction except for the side effect of drowsiness and constipation, which was positive. A significant constant term $(\alpha)$ indicates that other unmeasured factors considered by respondents, but not included in this experiment, influenced patient decision-making.

Inputting background characteristics into the model did not improve the model fit, nor were the associated $\beta$-parameters significant. The relative influence of cost on medication choice was not influenced by health care concession card status, private health insurance status, or work status.

The WTA for each factor is displayed in Table 4. Respondents were willing to accept high blood pressure, heart/kidney/liver problems as a side effect if compensated with up to $\$ 92$ per month. By contrast, respondents were willing to accept up to $\$ 14$ per month for a

Table 2 Factor Levels used in calculating odds ratios and predicted probabilities

\begin{tabular}{lllll}
\hline Factor & Glucosamine & $\begin{array}{l}\text { Acetaminophen } \\
\text { Sustained release }\end{array}$ & NSAID (selective, e.g. celecoxib) & NSAID (non-selective, e.g. ibuprofen) \\
\hline Pain Efficacy & 1 & 1 & 1 & 1 \\
Mode of Action & Slow OA & Quick & Quick & Quick \\
Dose Frequency & Three & Three & Once & Three \\
Treatment Schedule & Daily & Daily & Daily & Daily \\
Cost $(\$ A U)^{a}$ & $\$ 20$ & $\$ 12.00$ & $\$ 34.20$ & $\$ 20$ \\
Prescription & No & No & Yes & No \\
Side effects & Nil & Nil & High blood pressure, heart/kidney/ & Heartburn/reflux, stomach ulcers \\
\hline
\end{tabular}

${ }^{\mathrm{a}}$ The out-of-pocket costs represent those that would have been incurred th the time of the survey for non-healthcare concession cardholders. 
Table 3 Background characteristics

\begin{tabular}{|c|c|c|c|c|}
\hline & Responders $(n=188)$ & Missing (n) & All $(n=503)$ & Missing (n) \\
\hline Age (mean, SD), years & $62,8.5$ & 15 & 62,8 & 1 \\
\hline Gender $(n, \%)$, male & $84(48 \%)$ & 15 & $230(46 \%)$ & 1 \\
\hline Private Health Insurance (n, \%) & $108(62 \%)$ & 13 & $308(61 \%)$ & 0 \\
\hline Health Care Concession (n, \%) & $18(10 \%)$ & 13 & $56(11 \%)$ & 0 \\
\hline \multicolumn{5}{|l|}{ Co-morbidity with treatment $(n, \%)$} \\
\hline Hypertension or Heart Disease & $90(52 \%)$ & 15 & $213(45 \%)$ & 1 \\
\hline Ulcer or Stomach Disease & $13(7.5 \%)$ & 15 & $46(9 \%)$ & 1 \\
\hline Kidney or Liver Disease & $0(0 \%)$ & 15 & $4(1 \%)$ & 1 \\
\hline Symptom duration ( $\leq 5$ years) $(n, \%)$ & $92(52 \%)$ & 13 & $281(56 \%)$ & 0 \\
\hline WOMAC Pain (mean, sd) & $4.1(3.6)$ & 0 & $4.2(3.5)$ & 0 \\
\hline WOMAC Physical (mean, sd) & $15.9(14.0)$ & 0 & $17.0(13.3)$ & 0 \\
\hline Global assessment (mean (SD)) & $1.6(1.0)$ & 18 & $1.8(1.1)$ & 48 \\
\hline SF12 MCS (mean, SD) & $52.7(10.3)$ & 13 & $53.5(9.4)$ & 0 \\
\hline SF12 PCS (mean, SD) & $46.3(9.4)$ & 13 & $44.4(9.5)$ & 0 \\
\hline Glucosamine/chondroitin prior (n, \%) & $61(35 \%)$ & 13 & $154(31 \%)$ & 0 \\
\hline Current $<$ daily/when required medication use & $5(3 \%)$ & 13 & $38(8 \%)^{*}$ & 0 \\
\hline Adherence (study treatment) $<100 \%^{a}$ & $86(53 \%)$ & 3 & $N / A^{b}$ & $N / A^{b}$ \\
\hline Cost-related non-adherence ${ }^{a}$ & $19(10 \%)$ & 4 & $N / A^{b}$ & $N / A^{b}$ \\
\hline Physician Trust ${ }^{\mathrm{a}}$ (median, range) & $75(28-100)$ & 0 & $N / A^{b}$ & $N / A^{b}$ \\
\hline
\end{tabular}

* Sig $\mathrm{P}<0.05{ }^{\mathrm{a}}$ Measured in the MEDOS study only ${ }^{\mathrm{b}} \mathrm{N} / \mathrm{A}$ not applicable.

treatment that would only provide pain relief, in comparison to one that would slow OA.

The relative importance of the statistically significant factors is displayed in Table 4. The side effect of high blood pressure, heart/liver/kidney problems was most important, followed by out-of-pocket costs. Respondents were least concerned about the side effect of drowsiness and constipation.
Figure 1 [45] compares the relative likelihood of continuing GS, sustained-release acetaminophen, and selective and non-selective NSAIDs. In this figure, an odds ratio of 1 equates to an average utility $(\mathrm{U})$ of zero, implying that there is no preference (either positive or negative) to continue taking that medication. Assuming equivalent pain efficacy, GS as a disease-modifying agent, and no side effects with sustained-release acetaminophen, the relative likelihood of

Table 4 Discrete choice experiment results

\begin{tabular}{|c|c|c|c|}
\hline Factor & $\mathrm{OR}^{\mathrm{a}}(95 \% \mathrm{Cl})$ & $\mathbf{R I}^{\mathbf{a}}$ & WTA $^{a}(95 \% \mathrm{Cl})(\$ A U D)$ \\
\hline Pain Efficacy ${ }^{\text {b }}$ (decreasing) & $1.00(0.97-1.04)$ & $N / A^{a}$ & $0.08(0.04-0.11)$ \\
\hline Mode of action ${ }^{c}\left(\right.$ Slow $O A^{a}$ ) & $1.17(1.09-1.25)^{*}$ & 5 & $13.83(13.73-13.93)$ \\
\hline Dose frequency ${ }^{\mathbf{b}}$ (once/day) & $1.02(0.96-1.08)$ & & $0.58(0.42-0.75)$ \\
\hline Treatment Schedule ${ }^{c}$ (daily) & $0.90(0.89-0.9)^{*}$ & 4 & $4.11(3.92-4.29)$ \\
\hline Cost $^{b}$ (increasing) & $0.97(0.97-0.98)^{*}$ & 2 & $N / A^{a}$ \\
\hline Prescription $^{\mathrm{b}}$ (Yes) & $1.03(0.97-1.09)$ & $N / A^{a}$ & $1.00(0.83-1.16)$ \\
\hline \multicolumn{4}{|l|}{ Side effects } \\
\hline Drowsy/constipated $^{\mathbf{c}}$ & $1.55(1.48-1.62)^{*}$ & 6 & $18.06(17.90-18.23)$ \\
\hline Heartburn/reflux, stomach ulcers ${ }^{\mathbf{c}}$ & $0.76(0.75-0.78)^{*}$ & 3 & $10.67(10.55-10.78)$ \\
\hline High blood pressure, heart/kidney/liver problems ${ }^{c}$ & $0.09(0.08-0.11)^{*}$ & 1 & $90.50(89.38-91.62)$ \\
\hline Constant ${ }^{b}(a)$ & $N / A^{a}$ & $N / A^{a}$ & \\
\hline Model Fit Statistics & McFaddens $\rho 2$ adjusted & 0.37 & $\mathrm{AlC}^{\mathbf{a}}$ \\
\hline
\end{tabular}

*P $<0.001$.

a: OR Odds Ratio, RI Relative Importance, WTA Willingness to Accept, OA Osteoarthritis, N/A not applicable, AIC Akaike's Information Criterion.

b. Non-random parameter c: Random parameter. 


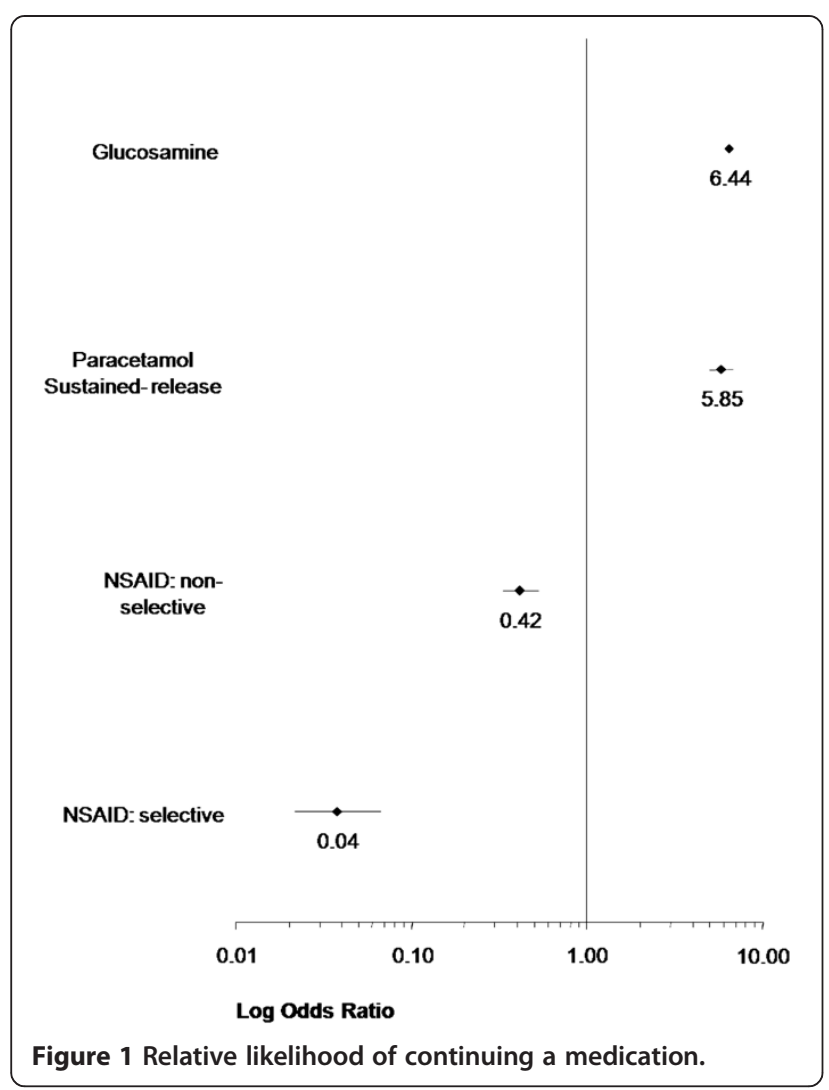

continuing with sustained-release acetaminophen taken regularly is positive and approximately equivalent to GS. By contrast, the relative likelihood of continuing a selective or a non-selective NSAID, taken regularly, is negative. The cost sensitivity analysis for GS (Table 5) reveals that the predicted probability of continued use of GS when provided without charge is $91.6 \%$, however when the cost rises to $\$ 50$ per month, the predicted uptake drops to $75 \%$.

\section{Discussion}

Using DCE, this study has assessed the factors that influence the decision to continue with a medication among a group of people with symptomatic OA. To the best of our knowledge, this is one of few DCEs assessing medication preferences nested within a clinical trial [46], and

Table $\mathbf{5}$ Cost sensitivity analyses for glucosamine sulfate

\begin{tabular}{cc}
\hline $\begin{array}{c}\text { Out of pocket monthly } \\
\text { cost (\$AUD) }\end{array}$ & $\begin{array}{c}\text { Predicted probability of } \\
\text { continued use (\%) }\end{array}$ \\
\hline 0 & 91.6 \\
10 & 89.3 \\
20 & 86.5 \\
30 & 83.2 \\
40 & 79.2 \\
50 & 74.6 \\
\hline
\end{tabular}

the first in an OA clinical-trial population. Such decisions underscore the concept of intentional medication non-adherence, which may influence the translation of clinical-trial results into practice.

This study has found that treatment factors, as opposed to respondent characteristics including self-reported pain levels and physical functioning, were driving adherence decisions. Preferences to continue with OA treatments were influenced by, in order of importance: the possibility of high blood pressure, heart/liver/kidney problems as side effects, out-of-pocket costs, the possibility of heartburn/ reflux, or stomach ulcers as side effects, treatment schedule (i.e.: daily versus when required), mode of action (slowing OA versus symptomatic pain relief) and the possibility of drowsiness or constipation as a side effect.

Perhaps surprisingly, treatment efficacy did not significantly influence patient choices in this study. However in contrast to previous DCE studies conducted among OA patients in observational settings [23,24,28], this study included additional factors related to treatment schedule as well as cardiovascular, liver and renal side effects. When treatment schedule and cardiovascular, liver, and renal side effects were taken into account, as in the present study, their influence over patients' treatment preferences then seem to dominate over considerations of treatment efficacy.

Assuming equal efficacy and GS as a disease-modifying agent, this study has found that the relative likelihood of continuing sustained-release acetaminophen and GS are positive and in contrast to NSAIDs. This disparity in predicted adherence was primarily driven by negative preferences expressed for cardiovascular, liver and renal side effects. This result may reflect an increased awareness of, or general aversion to, NSAID-related side effects through the recent and widely publicised removal of two NSAIDs, rofecoxib (2004) and lumaracoxib (2007), from the Australian market due to cardiovascular and hepatic toxicity, respectively [47,48].

However, the reduced likelihood of continuing NSAIDs compared to acetaminophen predicted from this study seems to be at odds with the high levels of self-reported use of NSAIDs in Australia by people with OA [3]. In light of the efficacy and improved safety of acetaminophen in OA compared to NSAIDs [3,5], our findings therefore reinforce the message that the uptake of guidelinesrecommended acetaminophen in practice would benefit from ensuring that patient medication decision-making is supported through the provision of explicit risk/benefit information.

Medication adherence within a clinical trial is typically higher than in observational settings [49]. For the LEGS study, as the treatment was provided free of charge, the level of medication adherence observed in the trial was predicted to be higher than would exist outside of the 
trial setting. This was demonstrated in the findings of the cost-sensitivity analysis: when provided free of charge as per study protocol, the predicted continuation of GS is around 91\%; however at the average current monthly price for GS (\$20), this figure dropped to below $80 \%$. Such rates of long-term adherence and their sensitivity to out-of-pocket costs will need to be factored into translation of the findings into policy and future economic evaluations.

The findings of this study must be viewed in light of its limitations. First, this study was conducted within a clinical-trial population, which may affect the generalisability of results. In particular, the self-reported adherence to medications and the proportion of participants using "when required" medications in this study was high. However, current medication use and self-reported adherence did not improve the model fit, suggesting that such preferences are formed independent of self-reported medication-taking behaviour.

Second, while discrete choice methods are widely used in health economics, an inherent limitation is that respondents are evaluating hypothetical medications; what respondents declare they will do may be quite different to what they would actually do if faced with the consequences of a choice. Forcing respondents to choose between medications may also be contrary to actual behaviour, particularly considering the over-riding influence of a prescriber's recommendations upon patient preventive treatment decisions [22,50]. To minimise such potential differences, measures were taken to design the hypothetical tasks to be realistic, for instance by centring levels of cost on current treatment costs and describing pain on the same scale used throughout the clinical trial. Encouragingly, in this study, trust in prescribers and the actual self-reported adherence did not influence the model results. Nonetheless, to investigate the relationship between relative preferences captured in this study and one's absolute preference to adhere to medications, future research incorporating the influence of the prescribers recommendation, for instance by allowing respondents to opt out of the non-adherent choice $[31,50]$, is warranted.

Finally, as the constant term $(\alpha)$ was significant, the factors included in this study do not explain all of the behaviour modelled. Further work is therefore needed to clarify which other factors are being considered in adherence decisions.

\section{Conclusions}

Osteoarthritis is a chronic condition incurring considerable costs to most health care systems. As with any chronic condition, non-adherence to the available pharmacological treatments is a problem that has the potential to impact on population health and expenditure. In the context of a clinical trial assessing therapy effectiveness, non-adherence has the potential to derail translation into clinical practice. By recognising that a component of this health behaviour is intentional and subject to rational choices, this study has characterised the complexity of medication-taking decisions for people with symptomatic OA that may lead to intentionally non-adherent behaviour, identifying the treatment factors driving such decisions. Such factors may be amenable to intervention such as strategic pricing. The salience of medication risks in such choices highlights the importance of providing appropriate risk/benefit information upon prescription. Cost was also a strong consideration in medication-taking decisions, a finding that ought to be acknowledged when interpreting clinical trial evidence for practice. Ultimately addressing these factors may be the way forward to realising the full potential health and economic benefits from the efficacious and safe use of osteoarthritis medications.

\section{Additional files}

\section{Additional file 1: LEGS participant eligibility criteria. \\ Additional file 2: Discrete Choice Experiment Model Form and} Analysis.

\begin{abstract}
Abbreviations
AIC: Akaike's information criterion; DCE: Discrete choice experiment;

GS: Glucosamine sulfate; LEGS: Long-term evaluation of glucosamine sulfate; LL: Log likelihood; MEDOS: Medication decisions in osteoarthritis study; MMNL: Mixed multinomial logit model; NSAID: Non-steroidal antiinflammatory drug; OA: Osteoarthritis; OR: Odds ratio; PCAS: Primary care assessment survey; WOMAC: Western Ontario and McMasters universities arthritis index; WTA: Willingness to accept.
\end{abstract}

\section{Competing interests}

The authors declare that they have no competing interests.

\section{Authors' contributions}

This work is a component of TL's doctoral research supervised by SJ and JB. $\mathrm{TL}, \mathrm{SJ}, \mathrm{MF}$ and JB have all made substantial contributions to the conception, design, acquisition, analysis and interpretation of the data, as well to the critical revision of the manuscript. All authors have read and approved the final manuscript.

\section{Acknowledgements}

The authors would like to acknowledge Maria Agaliotis and Lisa Bridgett from the Faculty of Health Sciences, University of Sydney, for their assistance with survey distribution and return.

\section{Author details \\ ${ }^{1}$ Faculty of Pharmacy, The University of Sydney, Camperdown, Sydney, Australia. ${ }^{2}$ The George Institute for Global Health, Camperdown, Sydney, Australia. ${ }^{3}$ St Vincent's Hospital, Darlinghurst, Sydney, Australia. ${ }^{4}$ Faculty of Medicine, The University of New South Wales, Kensington, Sydney, Australia. ${ }^{5}$ Faculty of Health Sciences, The University of Sydney, Lidcombe, Sydney, Australia. 'School of Medicine, The University of Sydney, Camperdown, Sydney, Australia.}

Received: 21 December 2012 Accepted: 23 April 2013

Published: 6 May 2013 


\section{References}

1. Woolf $A D$, Pfleger B: Burden of major musculoskeletal conditions. Bull World Health Organ 2003, 81:646-656.

2. Access Economics Pty Ltd: Painful realities: The economic impact of arthritis in Australia in 2007, A Report for Arthritis Australia. 2007. available online at: http://www.deloitteaccesseconomics.com.au/.

3. AlHW: Medication use for arthritis and osteoporosis. Arthritis series no. 11. Cat no. PHE 121. Canberra: Australian Institute of Health and Welfare (AlHW) 2010.

4. Carr A: Barriers to the effectiveness of any intervention in OA. Best Pract Res Clin Rheumatol 2001, 15:645-656.

5. Hochberg MC, Altman RD, April KT, Benkhalti M, Guyatt G, McGowan J, Towheed T, Welch V, Wells G, Tugwell P: American College of Rheumatology 2012 Recommendations for the use of nonpharmacologic and pharmacologic therapies in osteoarthritis of the hand, hip, and knee. Arthritis Care Res 2012, 64:465-474.

6. Wegman A, van der Windt D, van Tulder M, Stalman W, de Vries T: Nonsteroidal antiinflammatory drugs or acetaminophen for osteoarthritis of the hip or knee? A systematic review of evidence and guidelines. J Rheumatol 2004, 31:344-354.

7. Briggs A, Scott E, Steele K: Impact of osteoarthritis and analgesic treatment on quality of life of an elderly population. Ann Pharmacother 1999, 33:1154-1159.

8. MacLennan AH, Wilson DH, Taylor AW: The escalating cost and prevalence of alternative medicine. Prev Med 2002, 35:166-173.

9. Sibbritt D, Adams J, Lui CW, Broom A, Wardle J: Who uses glucosamine and why? A study of 266,848 Australians aged 45 years and older. PLoS One 2012, 7:e41540.

10. Black C, Clar C, Henderson R, MacEachern C, McNamee P, Quayyum Z, Royle $P$, Thomas S: The clinical effectiveness of glucosamine and chondroitin supplements in slowing or arresting progression of osteoarthritis of the knee: a systematic review and economic evaluation. Health Technol Assess 2009, 13:1-148.

11. Bruyere O, Reginster JY: Glucosamine and chondroitin sulfate as therapeutic agents for knee and hip osteoarthritis. Drugs \& aging 2007, 24:573-580.

12. About the PBS. http://www.pbs.gov.au/info/about-the-pbs.

13. Blamey R, Jolly K, Greenfield S, Jobanputra P: Patterns of analgesic use, pain and self-efficacy: a cross-sectional study of patients attending a hospital rheumatology clinic. BMC Musculoskelet Disord 2009, 10:137.

14. Cryer B, Luo X, Assaf AR, Sands G, Mardekian J: Persistence with nonselective NSAIDs and celecoxib among patients with gastroesophageal reflux disease and osteoarthritis or rheumatoid arthritis. Curr Med Res Opin 2011, 27:295-302.

15. Dominick KL, Baker TA: Racial and ethnic differences in osteoarthritis: prevalence, outcomes, and medical care. Ethn Dis 2004, 14:558-566.

16. Punchak S, Goodyer LI, Miskelly F: Use of an electronic monitoring aid to investigate the medication pattern of analgesics and non-steroidal antiinflammatory drugs prescribed for osteoarthritis. Rheumatology (Oxford) 2000, 39:448-449.

17. de Klerk E, van der Heijde D, Landewe R, van der Tempel H, Urquhart J, van der Linden S: Patient compliance in rheumatoid arthritis, polymyalgia rheumatica, and gout. J Rheumatol 2003, 30:44-54.

18. Salt E, Peden A: The complexity of the treatment: the decision-making process among women with rheumatoid arthritis. Qual Health Res 2011, 21:214-222.

19. Lehane E, McCarthy G: Intentional and unintentional medication nonadherence: a comprehensive framework for clinical research and practice? A discussion paper. Int J Nurs Stud 2007, 44:1468-1477.

20. Sale JE, Gignac M, Hawker G: How "bad" does the pain have to be? A qualitative study examining adherence to pain medication in older adults with osteoarthritis. Arthritis Rheum 2006, 55:272-278.

21. Milder TY, Lipworth WL, Williams KM, Ritchie JE, Day RO: "It looks after me": how older patients make decisions about analgesics for osteoarthritis. Arthritis Care Res (Hoboken) 2011, 63:1280-1286.

22. Milder TY, Williams KM, Ritchie JE, Lipworth WL, Day RO: Use of NSAIDs for osteoarthritis amongst older-aged primary care patients: engagement with information and perceptions of risk. Age Ageing 2011, 40:254-259.
23. Ratcliffe J, Buxton M, McGarry T, Sheldon R, Chancellor J: Patients' preferences for characteristics associated with treatments for osteoarthritis. Rheumatology (Oxford) 2004, 43:337-345

24. Fraenkel L, Bogardus ST Jr, Concato J, Wittink DR: Treatment options in knee osteoarthritis: the patient's perspective. Arch Intern Med 2004, 164:1299-1304.

25. Fraenkel L, Wittink DR, Concato J, Fried T: Informed choice and the widespread use of antiinflammatory drugs. Arthritis Rheum 2004, 51:210-214

26. Fraenkel L, Wittink DR, Concato J, Fried T: Are preferences for cyclooxygenase- 2 inhibitors influenced by the certainty effect? J Rheumatol 2004, 31:591-593.

27. Richardson CG, Chalmers A, Llewellyn-Thomas HA, Klinkhoff A, Carswell A, Kopec JA: Pain relief in osteoarthritis: patients' willingness to risk medication-induced gastrointestinal, cardiovascular, and cerebrovascular complications. J Rheumatol 2007, 34:1569-1575.

28. Hauber AB, Arden NK, Mohamed AF, Reed Johnson F, Peloso PM, Watson DJ, Mavros P, Gammaitoni A, Sen SS, Taylor SD: A discrete-choice experiment of United Kingdom patients' willingness to risk adverse events for improved function and pain control in osteoarthritis. Osteoarthritis Cartilage 2013, 21(2):289-297.

29. Lamiraud K, Geoffard P-Y: Therapeutic non-adherence: a rational behavior revealing patient preferences? Health Econ 2007, 16:1185-1204.

30. Laba $T L$, Brien JA, Jan S: Understanding rational non-adherence to medications. A discrete choice experiment in a community sample in Australia. BMC Fam Pract 2012, 13:61.

31. Bridges JF, Hauber AB, Marshall D, Lloyd A, Prosser LA, Regier DA, Johnson FR, Mauskopf J: Conjoint analysis applications in health-a checklist: a report of the ISPOR Good Research Practices for Conjoint Analysis Task Force. Value Health 2011, 14:403-413.

32. Lancsar $E$, Louviere J: Conducting discrete choice experiments to inform healthcare decision making: a user's guide. Pharmacoeconomics 2008, 26:661-677

33. Louviere JJ, Lancsar E: Choice experiments in health: the good, the bad, the ugly and toward a brighter future. Health Econ Policy Law 2009, 4:527-546.

34. Ryan M: A role for conjoint analysis in technology assessment in health care? Int J Technol Assess Health Care 1999, 15:443-457.

35. Ryan M, Gerard K: Using discrete choice experiments to value health care programmes: current practice and future research reflections. Appl Health Econ Health Policy 2003, 2:55-64.

36. Piette JD, Heisler M, Krein S, Kerr EA: The role of patient-physician trust in moderating medication nonadherence due to cost pressures. Arch Intern Med 2005, 165:1749-1755.

37. Safran DG, Kosinski M, Tarlov AR, Rogers WH, Taira DH, Lieberman N, Ware JE: The Primary Care Assessment Survey: tests of data quality and measurement performance. Med Care 1998, 36:728-739.

38. Henscher DA, Rose JM, Greene WH: Applied Choice Analysis: A Primer. UK: Cambridge University Press; 2005.

39. Louviere J, Henscher DA, Swait J: Stated choice methods: analysis and application. Cambridge: Cambridge University Press; 2000.

40. Ngene 1.0. http://www.choice-metrics.com/

41. Microsoft Corporation: Microsoft Office Excel 2003, Microsoft Office Professional Editions. 2003.

42. Buckland-Wright JC, Wolfe F, Ward RJ, Flowers N, Hayne C: Substantial superiority of semiflexed (MTP) views in knee osteoarthritis: a comparative radiographic study, without fluoroscopy, of standing extended, semiflexed (MTP), and schuss views. J Rheumatol 1999, 26:2664-2674.

43. Hole AR: A comparison of approaches to estimating confidence intervals for willingness to pay measures. Health Econ 2007, 16:827-840.

44. Hall J, Kenny P, King M, Louviere J, Viney R, Yeoh A: Using stated preference discrete choice modelling to evaluate the introduction of varicella vaccination. Health Econ 2002, 11:457-465.

45. Forest plots in excel software(Data sheet). Available at Please amend to www.evidencias.com.br.

46. Lancsar EJ, Hall JP, King M, Kenny P, Louviere JJ, Fiebig DG, Hossain I, Thien $F C$, Reddel HK, Jenkins CR: Using discrete choice experiments to investigate subject preferences for preventive asthma medication. Respirology (Carlton, Vic) 2007, 12:127-136. 
47. Therapeutics Goods Administration: Lumaracoxib (Prexige): Medicine recall. 2007. Available at http://www.tga.gov.au/safety/recalls-medicine-lumiracoxib-070810. $\mathrm{htm}$.

48. Therapeutic Goods Administration: Vioxx (Rofecoxib): Medicine Recall. 2004 Available at http://www.tga.gov.au/safety/recalls-medicine-vioxx-041001.htm.

49. Osterberg L, Blaschke T: Adherence to medication [see comment]. N Engl J Med 2005, 353:487-497.

50. Ryan M, Skatun D: Modelling non-demanders in choice experiments. Health Econ 2004, 13:397-402.

doi:10.1186/1471-2474-14-160

Cite this article as: Laba et al:: Patient preferences for adherence to treatment for osteoarthritis: the MEdication Decisions in Osteoarthritis Study (MEDOS). BMC Musculoskeletal Disorders 2013 14:160.

\section{Submit your next manuscript to BioMed Central and take full advantage of:}

- Convenient online submission

- Thorough peer review

- No space constraints or color figure charges

- Immediate publication on acceptance

- Inclusion in PubMed, CAS, Scopus and Google Scholar

- Research which is freely available for redistribution 